Cuadernos de Geografía • $104 \bullet 43-58 \bullet$ València 2020

JESÚS GARCÍA-ARAQUEa

\title{
MAPEOS COLABORATIVOS: OPORTUNIDAD PARA LA GEOGRAFÍA DE ACRECENTAR EL USO DE UNA VALIOSA HERRAMIENTA DE ANÁLISIS TERRITORIAL
}

\begin{abstract}
Resumen
La disciplina geográfica durante siglos ha destacado en el desarrollo y estudio de mapas, pero pudiera haberse quedado atrás en el uso de una fuente de información de eminente enfoque espacial, como es la cartografía colaborativa. No se trata de una herramienta novedosa, pero su uso se ha extendido en la última década y ha evolucionado acorde a nuevas tecnologías y métodos cartográficos, a la vez que se está viendo dominada por disciplinas sin relación con el análisis del territorio. Se pretende en el presente artículo indagar sobre la existencia de dicho distanciamiento por parte de la geografía y si los geógrafos han relegado los mapeos a un segundo plano. Con objeto de contrastar esta hipótesis se ha recurrido a dos fuentes. De una parte se ha examinado la evolución de esta herramienta a partir de un análisis bibliográfico, para descubrir que, ciertamente, ha avanzado pareja a una crítica a la cartografía oficial y un alejamiento del mundo académico. Seguidamente, se ha corroborado esta última apreciación analizando la rama de conocimiento de los organizadores de eventos estimados como relevantes en España.
\end{abstract}

PALABRAS ClAVE: cartografía participativa; geografía; mapeo colectivo; subjetividad.

a Universidad de Valladolid, Departamento de Geografía. Correo postal: Facultad de Filosofía y Letras, Plaza del Campus, s/n, Departamento de Geografía, 47011 Valladolid (España). Tfno. 983 186580. Email: jesus.garcia@uva. es. ORCID ID: https://orcid.org/0000-0002-4112-9404.

Fecha de recepción: 20/12/19. Fecha de aceptación: 27/3/20. 


\title{
COLLABORATIVE MAPPING: AN OPPORTUNITY FOR GEOGRAPHY TO INCREASE THE USE OF A VALUABLE TOOL FOR TERRITORIAL ANALYSIS
}

\begin{abstract}
Geographic discipline has stood out for centuries in the development and study of maps, but it could have lagged behind in the use of an eminent spatial approach information source, such as collaborative mapping. It is not a novel tool, but its use has extended in the last decade and has evolved according to new technologies and cartographic methods, while being dominated by disciplines unrelated to the analysis of the territory. It is intended in this article to inquire about the existence of such distancing by geography and whether geographers have relegated the mappings to the background. In order to test this hypothesis, two sources have been used. On the one hand, the evolution of this tool has been examined from a bibliographic analysis, to discover that, certainly, it has advanced to a criticism of official cartography and a departure from the academic world. Subsequently, this last assessment has been corroborated by analyzing the knowledge branch of the organizers of events estimated as relevant in Spain.
\end{abstract}

KEYwORDs: collective mapping; geography; participatory cartography; subjectivity.

\section{INTRODUCCIÓN, OBJETIVOS Y JUSTIFICACIÓN}

La elaboración de mapas en la forma tradicional es conocida por muchas denominaciones. Wood y Krygier (2009) aluden a cartografía profesional, académica u oficial para referirse a ella o, incluso simplemente "cartografía". Durante siglos, ha generado productos fundamentados en la ciencia, confeccionados por personas especializadas. Desde hace décadas, se extienden formas alternativas de hacer mapas. Algunas describen los territorios desde perspectivas discrepantes, desde visiones críticas o de denuncia, en busca de reflejar realidades sociales y procesos diferentes de los representados en los mapas convencionales, que incluso pretenden desafiar la ortodoxia.

Son englobadas comúnmente en el concepto de Cartografía Crítica, si bien Canosa y García (2017) reseñan otros seis nombres, cada uno con sus propias peculiaridades, como son la cartografía radical, disidente, indisciplinada, antagonista, social y contracartografía. Se encuentra extendida entre estas tendencias una predilección por rehuir el concepto de cartografía como muestra de discrepancia, aludiendo en muchos casos a "mapeo", como contraposición a "cartografía", para referirse a la práctica de hacer mapas alejados del plano oficial, a la vez que a "mapear", para referirse al hecho de "cartografiar".

La cartografía crítica en general pretende en muchos casos desvincularse de convecciones técnicas y académicas en el desarrollo de mapas. Autores tan prestigiosos como Lacoste (1977) sostienen que los mapas ortodoxos favorecen a grupos dominantes y reflejan relaciones de poder, al ser quienes controlan la representación cartográfica. Ciertamente hay muchos ejemplos de su utilización al servicio del poder. Sin ánimo de entrar en detalles, sirvan como muestra los mapas religiosos de la edad media (Morales, 2006) y los de la etapa colonial, en América, África y Asia, que determinan fronteras, mapean propiedades y cambian nombres (véase, entre otros, Crampton, 2010; Laxton, 2001). Asimismo, 
son muchas las críticas a la adulteración de la realidad que ofrecen las diferentes proyecciones, entre las que destaca, por su elevada utilización, la de Mercator, que muestra el hemisferio norte con superficie mayor de la real y el Sur más reducido (Martín, 2015).

Como respuesta a estas cuestiones, la cartografía crítica reivindica su papel bajo la idea de que la cartografía académica está muriendo al ignorar conocimiento territorial por no aceptar aquello que se aleja de sus directrices y restricciones. Por su parte, autores a favor de las nuevas tendencias opinan que los mapeos ofrecen mapas mas significativos y atractivos por carecer de limitaciones, de forma que permiten reinterpretar la superficie terrestre y aportar nuevo conocimiento de los territorios y de las personas que los habitan, así como de sus problemas sociales (Wood, 2003).

Entre los diferentes métodos existentes para elaborar estos mapas se encuentra aquel que requiere de la participación ciudadana, gracias a la colaboración de personas ajenas a la cartografía, que pueden, o no, ser residentes del lugar representado. Pueden desarrollar mapas desde cero, a partir de un papel en blanco, o reflejar reflexiones sobre mapas preexistentes. Asimismo, pueden realizarse sobre papel físico o a través de formatos digitales.

En un intento de romper con la tradición académica, quienes desarrollan cartografía o mapeos de forma colectiva recurren a un lenguaje propio. De esta forma, se denomina el proceso de creación de mapas mediante esta fórmula como mapping derivado de la unificación de los vocablos map y making (Wood, 2003), que ha sido adaptado al castellano a través del vocablo mapeo. Se encuentra extendida la costumbre de equiparar conceptualmente el proceso de elaboración y el producto resultante. Entre los términos más comúnmente utilizados se encuentran los de mapeo colectivo, colaborativo, participativo, social, mental o cognitivo. En gran cantidad de ocasiones se aplica simplemente el término mapeo, para hacer referencia tanto al mapa resultante, como a la realización de mapas de esta forma. Esto no quita para que también se aluda al producto final como mapa colectivo, participativo, etc...

Aunque esta forma de hacer mapas no es novedosa, su uso se ha extendido en los últimos años coincidiendo con la expansión de aproximaciones diferentes, en relación con la irrupción de nuevas y cada vez más complejas problemáticas sociales, en parte generadas por la crisis económica. Además, debe destacarse su avance acorde a nuevas tecnologías, con lo que a los tradicionales mapeos sobre papel físico se suman experiencias digitales de colaboración y representación, así como nuevas perspectivas y enfoques.

Más allá de discrepancias, es ampliamente aceptada la definición de "geografía" como una ciencia que representa, analiza, describe e interpreta la tierra (Ortega, 2000). Consecuentemente, los geógrafos han tenido supremacía en la elaboración, análisis e interpretación de mapas. Ante la imposibilidad de extenderse en el tema, cabe mencionar su estrecha relación con los mapas desde los primeros con base científica en Grecia, pasando, entre otros, por la cartografía medieval o decimonónica. Sin embargo, en los últimos años han perdido protagonismo, paralelamente a la incorporación de profesionales de otras ramas en la creación y manejo de cartografía. Por el momento, persiste una intrínseca asociación entre la geografía y la cartografía, y para los geógrafos los mapas se mantienen como herramientas esenciales, sin embargo, da la impresión de que no ocurre así con los mapeos colectivos.

Una gran cantidad de mapeos pretenden huir de lo académico, y tienen a la subjetividad como su razón de ser. Su desafío a la ortodoxia lleva a alejarse de la concepción de un espacio euclidiano, fun- 
damentado en valores absolutos, que se había mantenido durante siglos, para apostar por un contexto espacial relativo que, en palabras de Delgado (2003: 44) "abre un número infinito de nuevos mundos". Según Rekacewicz (2013) esa pretensión de alejarse de lo establecido genera representaciones rechazables por los geógrafos por ser inexactas y poco fiables, pero no por eso debe desdeñarse la información que proporcionan. Además, en la mano de los académicos está el transformar información subjetiva en trabajos objetivos y rigurosos. Si, como indica Higueras, el fin último de la geografía es comprender el territorio, no puede dejarse de lado ninguna contribución que aporte conocimiento, máxime si parte de visiones del territorio de quienes lo habitan.

Hay cartógrafos para quienes un mapa inexacto no tiene valor, hasta el punto que autores como Lynam o Letts entienden que los mapas anteriores al s. XX resultan inútiles y hasta "cómicos", entendiendo que son más "libros de pintura" que mapas (Crone, 1998). Sin embargo, insistiendo en ello, todo mapa aporta conocimiento del territorio, por lo que no se debe repudiar esta fuente de información a pesar de incorporar información subjetiva a los mapas que se crean.

Si se verifica la hipótesis de que la participación de los geógrafos es reducida, se pondría de manifiesto la existencia de un contratiempo para la disciplina geográfica debido a dos motivos: por la privación informativa que supone relegar de un instrumento de análisis territorial y por la incursión de otras disciplinas en la utilización de la más esencial de las herramientas geográficas. Con todo ello, debe aclararse que no se reclama la apropiación de esta técnica, que puede ser utilizada por quien lo considere oportuno, pero sí que la geografía se aproveche de ella y de la información que proporciona.

Pocos autores han estudiado los mapeos colectivos más allá de definirlos, justificar su utilización y mostrar indicaciones para su producción, y no se han detectado trabajos centrados en comprobar la participación de los geógrafos. Este asunto es tratado de forma indirecta por autores que prestan atención a la cartografía crítica, que en ocasiones han denunciado la poca presencia de geógrafos. En el caso nacional podemos observar a Canosa y García (2017), que denuncian un alejamiento mutuo entre los mapas críticos y la geografía y, anteriormente, Quirós (2001). Fuera de España destacan las manifestaciones de Rekacewcz (2013) y Cosgrove (2008). Sin embargo, ninguno se centra en la cartografía colaborativa, ni aporta datos de la desafección denunciada. Al no detectarse obras que hayan analizado la rama de conocimiento de procedencia de organizadores de los proyectos de mapeo colectivo, no se poseen referencias sobre el asunto estudiado con las que comparar los resultados de la investigación.

\section{Metodología Y FUeNTES DE INFORMACIÓN}

Con objeto de indagar en la participación de los geógrafos en procesos de interpretación del espacio a través de la cartografía colaborativa, para comprobar si es cierta su escasa implicación, se repara en los momentos clave en la evolución de esta técnica, desde su nacimiento hasta la actualidad, y se atiende a análisis efectuados sobre ella durante la última década, para terminar por comprobar el nivel de participación de los geógrafos españoles en la promoción de mapeos colectivos. 
Para este último examen se hace obligatorio establecer un filtro pues, considerando la facilidad con que pueden realizarse mapeados, especialmente desde la irrupción de las nuevas tecnologías, se hace imposible reparar en todos ellos.

De una parte se ha optado por investigar únicamente colectivos nacionales que hayan realizado varios proyectos y mapeos unitarios mencionados en la página web de la asociación Iconoclasistas (enlace disponible en tabla 1), por estimarse que se trata de una referencia global sobre el tema. La selección de estas fuentes se ha basado, para el primer caso, en escoger colectivos mencionados por autores que han estudiado y reflexionados sobre los mapeos colectivos, y aquellos que han adquirido mayor difusión, a partir del posicionamiento en el buscador Google al buscar palabras clave relacionadas con el asunto en cuestión, al tratarse, con diferencia, del buscador más utilizado en el mundo (Statista, 2019).

A partir de similar razonamiento, la página web Iconoclasistas ha sido seleccionada por ser un referente mundial, referido en multitud de los documentos tratados en este trabajo e, ineludiblemente, en todos los manuales que presentan instrucciones sobre cómo desarrollar un mapeo. Asimismo, disfruta de elevada difusión como consecuencia de su posición privilegiada en el buscador Google. Abarca mayoritariamente proyectos en América Latina, pero se ha estimado que, debido a su difusión, los proyectos españoles que aloja suponen una muestra representativa de lo que se está realizando en España. Esta metodología provoca que puedan quedar fuera trabajos significativos, pero establecer algún tipo de pauta discriminatoria se torna imprescindible.

Una vez estimados de esta forma los trabajos relevantes, se atenderá a la rama de conocimiento de los organizadores, lo que hace necesario de nuevo discriminar al existir proyectos sobre los que no se puede localizar información sobre ellos.

Para identificar proyectos y organizadores se recurre a revisión de hemeroteca y de referencias de autores que analizan el fenómeno, analizando sus obras y a rastreos por internet. Debe reseñarse que ante la tendencia a rehuir lo académico, alguna de las referencias bibliográficas consultadas puede tener procedencia un tanto informal.

\section{AnÁlisis eVolutivo de los MAPEOS COlectivos y ESTADO ACTUAl DE LA CUESTIÓN}

No se puede establecer un momento exacto a partir del cual se comienzan a elaborar mapas colectivos, puesto que exploradores y viajeros, muchos de ellos geógrafos, han considerado conocimientos de pobladores autóctonos durante siglos para elaborar mapas de territorios desconocidos. Según refleja Crone en su estudio de la historia de la cartografía (1998), esta metodología de trabajo fue habitual en etapas de exploración y descubrimiento, especialmente en América y, más aun si cabe, de África.

La inclusión de una perspectiva subjetiva y crítica en la confección de mapas elaborados colectivamente, que propiciaría el nacimiento de los mapeos, es situada por Páez (2013) en 1921, cuando grupos de artistas surrealistas, relacionados con el movimiento DADA, recorrieron ciudades francesas para describir sus impresiones mediante lo que denominaron mapas influénciales, que reflejaban su percepción espacial. Si bien no participan habitantes de los lugares descritos, es remarcable su carácter participativo y crítico. Los geógrafos mostraron distanciamiento con este tipo de experiencias, que se 
mantuvieron íntegramente relacionadas con el arte durante las siguientes cuatro décadas. Este método de trabajo evoluciona en los años 50, dando lugar a una corriente conocida como Internacional letrista, encabezada por el filósofo, escritor y cineasta Debord (1955), quien generó mapas psicogeográficos para entender la influencia del ambiente en las emociones y el comportamiento.

No sería hasta finales de los años sesenta cuando algunos geógrafos comienzan a incorporar este instrumento. La aparición de múltiples corrientes geográficas, como reacción al dominio del cuantitativismo en la investigación espacial, requirió de la aplicación de técnicas subjetivas de recopilación de información, mayoritariamente encuestas y entrevistas personales, pero también, en escasas ocasiones, mapeos colectivos. De una parte, mapas a partir de imágenes mentales, individuales y colectivas, elaborados por habitantes de los entornos investigados, se convierten en técnica esencial de análisis de algunos enfoques, como la geografía de la percepción y la humanista. En la primera, los mapas mentales, encuestas y entrevistas, permiten desarrollar mapas que identifican, principalmente, sentimientos de rechazo o identificación hacia el territorio. Como antecedente, de gran influencia sobre las corrientes geográficas mencionadas, el ingeniero y urbanista Lynch (1960) elaboró gráficos y mapas que definían paisajes urbanos a partir de diseños de pobladores, encuestas y entrevistas. El enfoque humanista recurre igualmente a mapas mentales, si bien su carácter antropocéntrico, con la idea de que cada ser humano es diferente como punto de partida, hace que en ocasiones se rehúya la realización colectiva al tender a apoyarse en valoraciones individuales.

Por otra parte, la geografía radical desarrolla mapeos desde finales de los años sesenta. Destaca la aportación de Bunge quien, después de ser referente del cuantitativismo, criticó el alejamiento de la realidad de esta perspectiva e impulsó la Geografía radical junto a Harvey. Ambos reclamaron la aplicación de conocimientos geográficos para conseguir cambios sociales, así como nuevas metodologías de recogida de información y análisis. Bunge elaboró mapas conjuntamente con pobladores de los barrios urbanos de metrópolis estadounidenses y canadienses entre 1968 y 1988, situando cartográficamente, entre otras cuestiones, crímenes o grafitis (Gómez, 1988).

En España, las perspectivas colectivas se desarrollaron con retraso respecto a otros países, según Estébanez (1982) por el arraigo del paradigma regional clásico. Durante la década de 1980 se elaboraron mapas sobre percepción urbana, atendiendo al espacio subjetivo, aplicando la metodología Lynch. Reseñan Boira y Reques (1992) los trabajos de Reques en Salamanca (1981), Oliva en Málaga (1982) y Vilagrasa y col. en Lérida (1983). A su vez, indican que los mapas resultantes, no pueden ser comparables ni representativos por su endeblez técnica y metodológica. Las críticas se suceden y autores de gran prestigio, como Capel (1988), definieron estos trabajos como meras descripciones, al entender que carecían de analítica y metodología replicables. Ante su subjetividad, tanto en España, como en el resto del mundo, los geógrafos se distancian a la vez que es ensalzado por artistas y militantes sociales procedentes de diferentes disciplinas, en muchos casos sin formación académica. Rekacewicz (2013) constata que a partir de 1990 comienzan a utilizarlo colectivos de lo más diversos, muchos sin relación con la cartografía, entre los que destacan estudiantes universitarios, arquitectos, antropólogos, sociólogos y militantes sociales, bajo el impulso de movimientos que reclamaban la participación ciudadana en la observación de sus problemas, cuestión marginalmente considerada hasta entonces. 
Paralelamente, desde mediados de 1990 se ha venido impulsando desde el plano político la implicación social y la participación de la población en procesos analíticos, que ha motivado la expansión de herramientas de análisis que posibilitan acercamiento a la realidad social. Muchos documentos europeos abogan por dicha aproximación y un papel activo de los ciudadanos, algunos de gran calado, como el Tratado fundacional de la UE (Consejo europeo, 1992), que promueve la descentralización en el diseño de políticas y mayor poder de entes locales o el Libro blanco de la Comisión Europea sobre gobernanza (2001). Ante estas propuestas en la elaboración de políticas sociales, los instrumentos de análisis que permiten participación han adquirido notoriedad. Como consecuencia, el mapeo se ha convertido en una herramienta de análisis al servicio de quienes pretenden conocer el territorio contando con la opinión de sus pobladores. En este contexto, diversidad de colectivos, desde asociaciones vecinales (AAVV) a ONG, han recurrido a la participación activa como herramienta de recogida de datos. Bajo esta idea de fomentar la gobernanza local, esta forma de recopilar datos ha tenido amplia aceptación en América latina en la última década, donde los más de cien mapeos colectivos impulsados en todo el mundo desde la asociación Iconoclasistas lo han convertido en referente mundial.

El alejamiento de los cartógrafos y geógrafos se ha incrementado a partir de la incorporación de nuevas tecnologías que, en palabras de muchos autores, entre otros Joliveau (2010), ha permitido una “democratización” de la cartografía. Conocido el fenómeno como neogeografía o neocartografía, están proliferando aplicaciones digitales que permiten participar de la creación, o crear individualmente, mapas con facilidad. Las opciones son cuantiosas, y no es intención extenderse de ellas. Valga con decir, como muestra de su diversidad, que abarcan desde la posibilidad de que cualquier persona trabaje en proyectos individuales, pasando por la colaboración en eventos puntuales o en otros a largo plazo, organizados por asociaciones diversas, $\mathrm{ONG}$ o empresas, que ofrecen plataformas propias o se sirven de otras previamente desarrolladas. Esta forma de trabajar ha extendido la utilización del concepto "Información Geográfica Voluntaria", para definir la producción de datos geoespaciales digitales por parte de ciudadanos sin conocimientos sobre el tema (Elwood, 2008). Haklay (2013) se refiere a ellos como desinformados y no capacitados, ya que puede tratarse de "cualquier persona, en cualquier lugar y en cualquier momento” (p. 2). Una opinión más positiva muestra Goodchild (2007) al reconocer que la información que proporcionan es económica y accesible, y la única disponible para lugares remotos.

Los eventos colaborativos se pueden orientar a denunciar cuestiones variadas, como pueden ser ambientales, sanitarias o urbanísticas, a la realización de bases cartográficas y, en ocasiones, para completar o corregir cartografía de empresas privadas. De esta forma, los resultados de las colaboraciones pueden ser de carácter libre o posesión de empresas, de forma que los autores carezcan de derechos sobre su aportación.

La literatura científica que ha analizado el fenómeno del mapeo durante la última década tiende a centrarse en la mencionada neocartografía, aunque persisten otras visiones. Como ocurre con la realización de mapeos, muchos autores de estos análisis son ajenos a la geografía. Se pueden distinguir tres orientaciones principales en estos estudios: las reflexiones genéricas que no profundizan en casos concretos, los análisis de una o varias experiencias concretas y los manuales con indicaciones sobre cómo realizar mapeos. En todo caso se reconoce una democratización de la geografía y, en una gran cantidad de ocasiones, se termina por reclamar el alejamiento de la ortodoxia y del mundo académico ante acu- 
saciones de parcialidad. Escasos trabajos pretenden lo contrario y anuncian dicha separación de forma constructiva para reclamar mayor relación. En esta situación se encuentran Canosa y García (2017) que denuncian la escasa atención de la geografía española y reclaman mayor participación. No obstante, destacan su vinculación con el arte y centran su trabajo en el análisis de mapas de un artista y poeta, licenciado en filología hispánica. Pueyo, Postigo, Arranz, Zúñiga, Sebastián, Alonso, López (2016) asumen falta de atención de los expertos en el espacio geográfico y alertan que puede llevar a consolidar lo que Palsky definió como cartografía indisciplinada y poner en entredicho el interés de esta herramienta. Para evitarlo, reclaman una respuesta desde la Geografía que permita aportar objetividad científica a la interacción entre expertos y habitantes. Gallar y Habegger (2006) creen necesario un trabajo conjunto entre investigaciones institucionales y militantes, pero de forma que esta colaboración no termine por significar una colonización de los movimientos sociales por parte de la Universidad.

Igualmente minoritaria es la postura que reclama que toda geografía debe alejarse del resto de ciencias para incorporarse a la lucha social, como solicita el colectivo "geobrujas" (González, Matamoros, Marchese, 2018).

Los estudios genéricos que tratan el tema sin profundizar en casos concretos mayormente tratan las geotecnologías y hacen constantes referencias a la democratización de la cartografía y su incidencia, aludiendo reiteradamente al distanciamiento respecto del mundo académico. Distingue Azócar (2016) dos cartografías paralelas, una oficial y otra participativa, ambas igual de válidas, pero que representan diferentes elementos de la realidad. Según Mateos (2013, p. 88) la diferencia entre ambas no se queda en la procedencia de los promotores y opina que el empoderamiento de los ciudadanos y su separación del mundo académico poseen enorme trascendencia social y tendrán "importantes consecuencias futuras que apenas alcanzamos a vislumbrar". Al respecto de las consecuencias, Jiménez (2011) opina que el cambio en la creación del espacio está transformando las relaciones de poder.

De esta forma, sin atisbo de dudas sobre el proceso de empoderamiento de las herramientas de representación del territorio por parte de los ciudadanos, puesto de manifiesto por los estudios genéricos sobre el tema, al igual que efectúan las otras dos formas de analizarlo que se expondrán a continuación, se han detectado tres discursos en relación con el tema:

Democratización de la
Geografía $\left(G^{a}\right)$

Separación de la $\mathrm{G}^{\mathrm{a}}$ del mundo académico para incorporarla a la lucha social

Desarrollo de dos $\mathrm{G}^{\text {as }}$ paralelas, dentro y fuera del mundo académico

En cuanto a recopilaciones y descripciones españolas de mapeos llama la atención el trabajo de Padrón Alonso (2011), que describe decenas de mapas críticos, con 23 contribuciones nacionales, y menciona seminarios, encuentros y plataformas relacionadas con el tema. Además, se refiere a otros catálogos similares fuera de España, como los de Harmon (2004), Bhagat y Mogel (2008) y Gayle y Harmon (2009). Hace mención a los reparos de la esfera académica y a la ausencia de estudios por considerarlo un producto "ligero". Entre las compilaciones españolas, es también reseñable, tanto por la recopilación efectuada como por su reprobación de la geografía tradicional, la de De Soto (2014), 
que aúna 34 mapas relacionados con el movimiento social "15M". Gran cantidad de ellos son de carácter colectivo y fueron creados mediante aplicaciones informáticas. Se refiere a todos como "fuera de la disciplina geográfica” y aclara que están elaborados por activistas sociales, hackers, arquitectos y urbanistas sin relación con la cartografía.

Esta actitud es habitual en las recopilaciones o análisis de experiencias que realizan asociaciones organizadoras de eventos. Sin abundar en estas asociaciones, dado que serán mencionadas en el apartado empírico, se puede aludir a Ecologistas en Acción o Médicos del Mundo, que reclaman la consabida democratización en la confección de mapas en sus textos. No obstante, es preciso hacer referencia a un colectivo que sobresale sobre los demás, y que se muestra extremadamente crítico con la convencionalidad, que es Iconoclasistas. Creado en Argentina en 2006 por una profesora de Ciencias Sociales y un artista gráfico, en su página web reseñan más de cien proyectos organizados por todo el mundo. Su reivindicación de una Geografía paralela alejada de lo académico, es constante.

En las exposiciones y/o análisis de una única experiencia la tendencia a cuestionar la geografía tradicional es más reducida y algunos autores se limitan a exponer los mapeos realizados sin entrar en reflexiones sobre el asunto. Es el caso, entre otros, de Ramos (2018), que recurre a la colaboración para señalar conflictos territoriales, o de Ares y Risler (2016) en los mapeos realizados en Santa María la Ribera, México, detallados en un documento que no entra en disquisiciones. Pero también existen otros que insisten en reclamaciones anti-objetividad, como la descripción de un estudio en Pelotas, Brasil, de Tetamanti y Rocha (2016), que aboga por una investigación-intervención con trabajos aplicables a procesos de mejora del territorio, justificando esta idea en la necesidad de huir de la imparcialidad para conocer el territorio. Estos autores afirman que la cartografía social "es un mapa festivo y aparentemente caótico, porque es dinámico y vive; en contrapartida con el solitario mapa de los institutos geográficos” (p: 103).

Los escasos manuales que muestran pautas de elaboración también critican generalmente los procesos tradicionales. Sin ánimo de extenderse en un asunto ya tratado, únicamente mencionar que es la postura del manual de mayor difusión y profundización, elaborado por Iconoclasisistas, que presenta y describe mapeos y otras posibilidades participativas para recabar información, además de los pasos a seguir (Ares, Risler, 2015). Con afinidad de ideología y proclamas, es reseñable la participación del Ministerio español de Educación, Cultura y Deporte en la guía didáctica de Lafuente y Horrillo (Coord., 2017), que reclama un conocimiento más allá de lo académico y de instituciones "canónicas", así como la expansión de enfoques diferentes para obtener información. Bajo la premisa de que es una herramienta válida de planeamiento urbano, incorporan conceptos e iniciativas de lo que denominan "otra forma de hacer las cosas" (p. 2) para recabar información aplicable a propuestas en el ámbito del urbanismo, e indican que todo mapeo es una acción crítica. Igualmente, entienden la necesidad de renovar las técnicas para obtener información Alberich, Arnanz, Basagoiti, Belmonte, Bru, Espinar, García, Habegger, Heras, Hernández, Lorenzana, Martín, Montañés, Villasante, Tenze (2009). Tras seguir un centenar de procesos participativos para la elaboración de su documento, abogan por metodologías de investigación-intervención que promuevan transformaciones sociales. De forma similar, la descripción de la metodología para mapear activos y recursos en salud de ámbito local de Médicos del Mundo de Botello, Palacio, García, Margolles, Mariano, Nieto, Cofiño (2012) entiende la necesidad de asociar la información obtenida y la acción. 
Tabla 1. Rama de conocimiento de miembros de colectivos promotores de mapeos colectivos. Fuente: elaboración propia.

\begin{tabular}{|l|l|l|}
\hline Colectivo / asociación & $\begin{array}{c}\text { Rama de conocimiento de } \\
\text { organizadores }\end{array}$ & \multicolumn{1}{|c|}{ Referencia } \\
\hline Asociaciones vecinales & Total heterogeneidad & Alberich (2007) \\
\hline CarTac & $\begin{array}{l}\text { Activismo social / } \\
\text { Arquitectura-urbanismo / } \\
\text { política }\end{array}$ & Padrón (2011); Canosa, García (2017) \\
\hline Geoinquietos & Geografía & http://geoinquietos.org/ \\
\hline GEOT & Geografía & Pueyo, Postigo, Sebastián, Zúñiga (2014) \\
\hline Hackitectura & Arquitectura / informática & Padrón (2011); Canosa, García (2017) \\
\hline Iconoclasistas & $\begin{array}{l}\text { Ciencias sociales / Arte } \\
\text { gráfico }\end{array}$ & https://www.iconoclasistas.net/ \\
\hline Idensitat & Arte & Padrón (2011); Canosa, García (2017) \\
\hline Mapeado colaborativo & $\begin{array}{l}\text { Arquitectura / Ingeniería } \\
\text { industrial / informática / }\end{array}$ & https://mapcolabora.org/ \\
\hline Geografía & Medicina / enfermería & http://madridsalud.es/mapeo-inicio/ \\
\hline Médicos sin Fronteras & Medicina / enfermería & https://www.missingmaps.org/es/ \\
\hline Meipi Asturias & Arquitectura / informática & $\begin{array}{l}\text { http://www.laboralcentrodearte.org/en/education/ } \\
\text { encuentro-internacional-de-cartografia-ciudadana }\end{array}$ \\
\hline $\begin{array}{l}\text { Observatorio Deuda en } \\
\text { la Globalización }\end{array}$ & $\begin{array}{l}\text { Activismo social } \\
\text { (Ecologistas en acción) }\end{array}$ & https://www.ecologistasenaccion.org/?p=22684 \\
\hline Paisaje transversal & Arquitectura & https://paisajetransversal.com/ \\
\hline Rotorr & Arte visual / diseño gráfico & Padrón (2011); Canosa, García (2017) \\
\hline
\end{tabular}

\section{AnÁlisis de proyectos españoles ReleVAntes}

Parece claro el intento de dejar de lado al mundo académico, pero queda por saber si se ha conseguido. Los colectivos más relevantes que han elaborado mapas colaborativos en España son de lo más diversos. Observando una recopilación de colectivos y asociaciones estimados como destacados (Tabla 1), se detectan pocos casos en los que participan personas relacionadas con la Geografía o cartografía.

Como representantes de esta disciplina académica, nos encontramos en esta recopilación con el colectivo Geoinquietos y el Grupo de Investigación GEOT. Geoinquietos está conformado por diferentes grupos informales que se reúnen para debatir y compartir experiencias sobre Geografía. Algunos de estos grupos han puesto en marcha iniciativas de mapeo. Relacionados con Geoinquietos, "mapeado 
colaborativo" se establece como un grupo de trabajo especializado en cartografía colaborativa, que ha organizado eventos utilizando la herramienta OpenStreetMap. Por su parte, en GEOT siguen una dinámica diferente. Desde hace una década, este Grupo de Investigación de la Universidad de Zaragoza ha llevado a cabo multitud de mapas con colaboración ciudadana. Tras efectuar diversos trabajos de este modo, expusieron una recapitulación de sus resultados en una exposición celebrada en el Centro Ambiental del Ebro en 2014, con la finalidad de acercar su producción a los vecinos y promover un modelo de gobernanza participativo (Pueyo, Postigo, Sebastián, Zúñiga, 2014).

Como paradigma de la disparidad de integrantes de una asociación, nos encontramos con las AAVV, hasta el punto de que no poseen cabezas visibles y no se pueden establecer ramas de conocimiento, pues cambian constantemente de dirigentes y miembros. No obstante, no deben obviarse estos trabajos, precisamente por este motivo. Muchos autores han constatado la heterogeneidad de sus miembros y dirigentes, como Alberich (2007), que distingue la existencia de amplios rangos de edad, condición socioeconómica, nivel de estudios, motivaciones...

Como muestra de los trabajos desarrollados en este tipo de asociaciones, puede destacarse, debido a su elevada repercusión, la utilización de esta herramienta por parte de AAVV madrileñas, que diagnosticaron sus barrios en el marco de los planes de barrio del Ayuntamiento. Entre otros, la AV Puente de Vallecas, efectuó diagnósticos espaciales participados entre 2015 y 2016, cuyos resultados aportaron información para redactar un plan integral de regeneración urbana ${ }^{1}$.

Llama la atención la persistencia de la relación con el arte, por medio de colectivos como Rotorr e Idensitat, mencionados y analizados en el catálogo de Padrón (2011) y por Canosa y García (2017). Asimismo, se mantiene una notable relación con la arquitectura, especialmente por parte de una disciplina directamente relacionada, como es el urbanismo, que ya fue reseñada por Rekacewicz (2013).

Más reciente es la implicación del área de la salud. Médicos sin Fronteras, con colaboración de las divisiones de Cruz Roja en Reino Unido y Estados Unidos, pusieron en marcha Missing maps en 2014, inicialmente para cartografiar colectivamente partes del mundo vulnerables a crisis humanitarias. Recientemente están organizando eventos virtuales masivos en países desarrollados, como el Mapatón humanitario 2019 en España, para el que colaboró el Departamento de Geografía de la Universidad de Zaragoz $a^{2}$. Por su parte, Médicos del Mundo han efectuado procesos por ciudades españolas para localizar activos en salud para conocer los bienes de la comunidad a través de su participación.

El colectivo Iconoclasistas merece especial atención y un capítulo aparte, pues todo lo referido en su página web adquiere gran repercusión. Procedentes del mundo de la comunicación, titulados en arte gráfico y ciencias sociales, sus fundadores no han participado directamente de mapeos en España, pero han impulsado varios y participado en talleres y seminarios. Entre los nueve mapeos españoles que ha promovido (Tabla 2), únicamente en dos se ha detectado la presencia de geógrafos: en la Facultad de

1 Véase: https://aavvmadrid.org/proyectos/planes-de-barrio/ y https://www.paisajetransversal.org/2015/12/mapeo-colectivo-puente-de-vallecas-regeneracion-urbana-integrada-participativa.html

2 Véase: https://www.msf.es/mapaton-la-cartografia-herramienta-humanitaria 
Historia de Vigo, cuya promotora además tiene formación en arqueología, y en Bilbao en 2018, donde uno de los cuatro organizadores posee formación en geografía e historia.

En la promoción del resto de eventos se mantiene el predominio del mundo del arte. El más reciente fue llevado a cabo en 2019 por el colectivo Enmedio, que cuenta entre sus filas con diseñadores, fotógrafos, cineastas y artistas, que justifican este trabajo por la escasa conexión entre acción política y arte. Destaca también entre los nueve proyectos nacionales la presencia de activistas sociales de asociaciones que luchan por mejorar la calidad de vida de los ciudadanos de espacios urbanos. A este respecto llama la atención como todos los mapeos españoles son de carácter urbano, mientras que la mayoría del centenar referido en Iconoclasistas, fueron efectuados en zonas rurales.

Tabla 2. Rama de conocimiento de organizadores de mapeos promovidos por Iconoclasistas en España. Fuente: elaboración propia.

\begin{tabular}{|l|l|l|}
\hline \multicolumn{1}{|c|}{ Ciudad de realización } & \multicolumn{1}{|c|}{ Fecha } & \multicolumn{1}{c|}{ Rama de conocimiento de organizadores } \\
\hline Barcelona & 2009, mayo & Técnico medioambiental / Activismo social \\
\hline A Coruña & 2009 & Estudiantes de arquitectura / Activismo social \\
\hline Málaga & 2010, marzo & Arte / Activismo social \\
\hline Vic & 2011, julio & Arte contemporáneo \\
\hline Barcelona & 2014, octubre & Arquitectura y filosofía / Arte y política \\
\hline Vigo & 2014, septiembre & Arqueóloga y geógrafa \\
\hline San Sebastián & 2016, mayo & Comunicación audiovisual \\
\hline Bilbao & 2018, noviembre & $\begin{array}{l}\text { Periodismo / sociología / docencia en Educación / } \\
\text { Geografía e historia }\end{array}$ \\
\hline Barcelona & 2019, abril & Profesionales de la imagen / arte \\
\hline
\end{tabular}

\section{Conclusiones}

Una mirada al progreso de los mapeos colectivos, desde sus primeros pasos hasta el actual modelo basado en la tecnología, así como al análisis de los organizadores de proyectos españoles de la última década, pone de manifiesto un progresivo distanciamiento de esta técnica por parte de una gran parte de los geógrafos. Asimismo, se ha observado que la Geografía no ha prestado atención al asunto hasta finales de los años 1960. Debe dejarse claro que esto no significa que se hayan desligado por completo, pues interesantes trabajos indican que no ha ocurrido, pero, lamentablemente, son pocos, y esta herramienta es mayoritariamente utilizada por otros colectivos.

La prevalencia de su utilización por parte de activistas sociales se muestra clara, al igual que la perseverancia en la participación de los artistas, asunto, este último, que no debiera antojarse llamativo, al haber sido los mapas asimilados con el arte durante siglos. Precisamente en la intromisión de otros actores puede encontrarse una motivación de la escasa participación constatada aquí de los geógrafos en mapeos colectivos. Siguiendo, en parte, a Wood (2003), se intuye que el alejamiento de las conven- 
ciones académicas supone una amenaza a la profesión y su prestigio por cuanto facilita la elaboración de mapas, y cualquier persona puede generarlos sin conocimientos cartográficos. La amenaza se ha agudizado con el paso del tiempo, dadas las facilidades que proporciona la neogeografía para desarrollar mapas de forma colectiva, con lo que cabe predecir que la situación persistirá. De esta forma, se intuye que la situación es asimilable para todo tipo de mapa crítico que rechace las normas cartográficas, no solo para los elaborados colectivamente, tratados en este documento, ya que, igualmente, supone una amenaza el que pueda realizarlo cualquier persona sin conocimientos. Directamente relacionado con un desprestigio de la profesión se encuentra el rechazo a la geografía euclidiana, que identifica un único espacio absoluto, que promueve la perspectiva relativa de los mapeos colaborativos y la consiguiente posibilidad de elaborar infinidad de mapas de un mismo espacio.

En cuanto a la rivalidad descubierta, llama la atención la reciprocidad de opiniones entre el ámbito académico y no académico. Ambos alegan un argumento similar para rechazar a sus "rivales". Los especialistas en cartografía tienden a rechazar los mapeos por requerimiento de una mayor objetividad, al opinar que son totalmente subjetivos. Mientras tanto, desde el activismo social se reclama esta herramienta y un alejamiento de los mapas académicos bajo el pretexto de que son elementos poco objetivos, con intencionalidad estratégica subjetiva y al servicio del poder.

El reclamo de objetividad por parte de los académicos se debe al uso de información subjetiva para crear los mapas, pero no por ello el resultado debe ser subjetivo, si se tratan los datos obtenidos con rigurosidad científica. En algunas de las experiencias nombradas se ha detectado el seguimiento de un estricto tratamiento científico, entre otras, en las desarrolladas por Médicos del Mundo, que siguen una exhaustiva metodología de trabajo, que ha sido replicada en diversas ciudades españolas.

En todo caso, se percibe que una gran cantidad de mapeos, incluso algunos creados desde la geografía, pretenden criticar a los mapas convencionales, estableciéndose como una crítica y no como una forma de obtener datos. Casi de forma generalizada, realizar un mapeo es considerado como un acto de rebeldía, como una crítica al poder establecido, a lo oficial y a lo académico. Se establece en muchos casos su finalidad en efectuar algún tipo de denuncia y no únicamente en generar conocimiento. Sin embargo, se opina que la crítica debiera provenir de los resultados del mapeo, y no establecer el uso de la propia herramienta en sí misma como una crítica. Los resultados de los mapeos pueden ser usados de forma crítica, pero no parece constructivo que el mero hecho de desarrollar un mapeo colectivo sea considerado una reprobación, como así se ha observado que ocurre.

Una mayor utilización por parte del ámbito académico podría alejar estas consideraciones establecidas por otros ámbitos, pues, con el debido paso del tiempo, permitiría establecer su concepción como una herramienta más y alejar su correspondencia con la rebeldía y con los relatos críticos.

Su validez como herramienta de análisis territorial ha quedado clara a lo largo de toda la exposición anterior, por cuanto no se puede negar que un mapeo no hace más que representar, analizar, describir e interpretar la tierra; retrotrayendo esta descripción a una definición de geografía ampliamente aceptada, como es la establecida por Ortega Valcárcel en su excelsa obra "Los horizontes de la Geografía" (Higueras, 2003). Sin lugar a dudas, un mapeo aporta conocimiento y datos, algunos de los cuales no pueden obtenerse de otro modo que no sea con un acercamiento a la realidad investigada. 
Indudablemente, no se pretende reclamar la exclusividad de la técnica del mapeo para la Geografía, pues la situación podría asimilarse, por ejemplo, a un intento de apropiación de las entrevistas, encuestas o del cálculo de probabilidades. No obstante, se recomienda que los geógrafos utilicen en mayor medida una fuente de información territorial con una sobresaliente capacidad para proporcionar información, reiterando lo dicho, alguna de la cual no podría llegar a obtenerse de otra manera.

\section{REFERENCIAS}

Alberich, T (2007): Asociaciones y movimientos sociales en España. Revista de estudios de juventud, 76, 71-89.

Alberich, T.; Arnanz, L.; Basagoiti, M.; Belmonte, R.; Bru P.; Espinar, C.; García, N.; Habegger, S.; Heras, P.; Hernández, D.; Lorenzana, C.; Martín, P.; Montañés, M.; Villasante, T.; Tenze, A. (2009): Metodologías participativas. Manual. Madrid. Observatorio Internacional de Ciudadanía y Medio Ambiente Sostenible (CIMAS).

Ares, P. y J. Rizler (2015): Manual de mapeo colectivo: recursos cartográficos críticos para procesos territoriales de creación colaborativa. Buenos Aires. Tinta Limón.

Ares, P. y J. Rizler (2016): Taller de mapeo colectivo Santa María la Ribera. InSite Casa Gallina, 13/18, $1-47$.

Azócar P. (2016): Nuevas prácticas cartográficas: democratización de la cartografía mediante geotecnologías y su impacto en el desarrollo local. Revista estudios políticos estratégicos, 4(2), 54-71.

Botello, B.; Palacio, S.; García, M.; Margolles, F.; Mariano, H.; Nieto, J.; Cofiño, R. (2012): Metodología para el mapeo de activos de salud en la comunidad. Gaceta Sanitaria, 27(2), 180-183.

Canosa, E. y A. García (2017): Cartografías críticas de la ciudad. Treballs de la Societat Catalana de Geografía, 84, 145-160.

Capel, H. (1988): Filosofía y ciencia en la Geografía contemporánea: una introducción a la Geografía. Barcelona. Barcanova.

Comisión Europea (2001): Libro blanco sobre la gobernanza. Bruselas. Diario Oficial C 287 de 12-10. 2001, COM 428, de 25 de julio de 2001.

Consejo Europeo (1992): Tratado de la Unión Europea. Maastrich. Diario Oficial C 191 de 29-07-1992, 0001-0110.

Cosgrove, D. (2008): Cultural cartography: maps and mapping in cultural geography. Annales de géographie, 2008/2-3 660,159-178.

Crampton, J. (2010): Mapping: Critical Introduction to Cartography and GIS. Hoboken, United States. Wiley-Blackwell.

Crone, G. (1998): Historia de los mapas. Madrid. Fondo de cultura económica de España. Madrid.

Debord, G. (1955): Introduction à une Critique de la Géographie Urbaine. Les Lèvres Nues, 6, 1-10.

Delgado, O. (2003): Debates sobre el espacio en la geografía contemporánea. Bogotá. Universidad Nacional de Colombia. 
De Soto, P. (2014): Los mapas del 15M: el arte de la cartografía de la multitud conectada. Barcelona. Papers $\&$ productions.

Elwood, S. (2008): Volunteered geographic information: future research directions motivated by critical, participatory, and feminist GIS. GeoJournal, 72, 173-183.

Estebánez, J. (1982): La geografía humanística. Anales de geografía de la Universidad Complutense, 2, 11-34.

Gallar, D. y S. Habegger (2006): Nuevos retos para los movimientos sociales. Memoria de XI jornadas internacionales sobre metodologías participativas, 14-16 de marzo 2006. CIMAS, 14-16.

Gómez, J. (1988): Las expediciones geográficas radicales a los paisajes ocultos de la América urbana, en Gómez, J. y Et Al. Viajeros y paisajes, Madrid, Alianza, 151-174.

Goodchild (2007): Citizens as sensors: the world of volunteered geography. GeoJournal, 69, 211-221.

González, H.; N. Matamoros y G. Marchese (2018): «Subvertir la cartografía para la liberación». Revista de la Universidad de México, julio 2018, 40-43.

Haklay, M. (2013): Neogeography and the delusion of democratisation. Environment and Planning A, 45(1), 55-69.

Higueras, A. (2003): Teoría y método de la geografía: introducción al análisis geográfico regional. Zaragoza. Prensas universitarias.

Horrillo, P. y A. Lafuente (Coord.) (2017): Como hacer un mapeo colectivo. Madrid. La aventura de aprender.

Jiménez, D. (2011): La Neo-geografía: cambios y permanencias en el ciber-espacio. Revista universitária de treballs académics, 3, 1-20.

Joliveau, T. (2010): La géographie et la géomatique au crible de la néogéographie. Tracés Revue de Sciences humaines, hors série 0, 227-239

Wood, D.; Krygier, J.B. (2009): “Map,” "Protest Maps,” “Critical Cartography,” "Map Types”. En: Kritcin, R.; Thrift, N. (Coord.), Encyclopedia of Human Geography. Elsevier. New York.

Lacoste, Y. (1977): La geografía: un arma para la guerra. Barcelona. Anagrama.

Laxton, P. (2001): The new nature of maps, essays in the history of cartography. Baltimore. JHU Press.

Lynch, K. (1960): La imagen de la ciudad. Massachusetts. The Massachusetts Institute Technology Press: (2008) Barcelona. Gustavo Gili.

Martin, J (2015): Historia de las proyecciones cartográficas. Madrid. Centro nacional de información geográfica.

Mateos, P. (2013): Geovisualización de la población: nuevas tendencias en la web social. Investigaciones geográficas, 60, 87-100.

Morales, M. (2006): La evolución de los mapas a través de la historia. Mapping, 110, 52-72.

Ortega, J. (2000): Los horizontes de la geografía. Barcelona. Editorial Ariel.

Padrón, D. (2011): Prácticas cartográficas antagonistas en la Época Global. Catálogo de mapas críticos. Proyecto de Máster, Universitat de Barcelona. Recuperado a partir de http://www.dianapadronalonso. $\mathrm{com} / \mathrm{p} /$ publications.html 
Paez, R. (2013): Derivas urbanas: la ciudad extrañada. Revista Indexada de Textos Académicos, 1, 120. 129.

Pueyo, A.; Postigo, R.; Sebastián, M.; Zúñiga, M. (2014): Zaragoza Mapa a Mapa: una herramienta para el ciudadano. XVI Congreso Nacional de Tecnologías de la Información Geográfica. Alicante. Universidad de Alicante, 856-864.

Pueyo, A.; Postigo, R. Arranz, A.; Zúñiga, M.; Sebastián, M.; Alonso, M. P.; López, C. (2016): La cartografía temática: una herramienta para la gobernanza de ciudades. Aportaciones de la Semiología Gráfica Clásica en el Contexto de nuevos paradigmas geográficos. Revista de Estudios Andaluces, 33(1), 84-110.

Quirós, F. (2001): Representación del espacio geográfico para su conocimiento, interpretación y gestión. Universidad de Oviedo, Actas del XVII Congreso de Geógrafos Españoles, 29-39.

Ramos, O. (2018): Mapeo y análisis espacial de conflictos territoriales para el programa de desarrollo con enfoque territorial del Catatumbo. Proyecto de Grado, Universidad Distrital de Bogotá, Repositorio. Recuperado a partir de http://repository.udistrital.edu.co/handle/11349/13980

Rekacewicz, P. (2013): Cartographie radicale. Monde Diplomatique, feb. 2013, p.15.

Reques P. y J. Boira (1992): Balance crítico de dos décadas de geografía de la percepción en España. Universidad de Zaragoza, Actas del V Coloquio de Geografía Cuantitativa, 341-356.

Statista (2019): Google, datos estadísticos. Consulta online el 2-9-19 en https://es.statista.com/temas/3540/google/

Tetamanti, J. y E. Rocha (2016): Cartografía social aplicada a la intervención en barrio Dunas, Brasil. Revista Geográfica de América Central, 2-57, 97-128.

Wood, D. (2003): Cartography is dead. Cartographic perspectives, 45, 4-7.

Cómo citar este artículo:

García-Araque, J. (2020). Mapeos colaborativos: oportunidad para la geografía de acrecentar el uso de una valiosa herramienta de análisis territorial. Cuadernos de Geografía, 104, 43-58.

https://doi.org/10.7203/cguv.104.16325

\section{(c) $(1) \Theta \Theta$}

Este obra está bajo una licencia de Creative Commons Reconocimiento-NoComercial-SinObraDerivada 4.0 Internacional. 